\section{Ultraviolet vision in lizards}

SIR - There is evidence that each of the main vertebrate groups can detect ultraviolet light ${ }^{1}$, but, in contrast to the situation in invertebrates, the role and/or importance of ultraviolet vision in vertebrates is under debate ${ }^{1}$. One sug gestion is that ultraviolet vision may be important in communication, but evidence to support this function is limited to the fact that the bodies of some fish and birds are highly reflective in ultraviolet light ${ }^{2,3}$. We have been studying five closely related species of anoline lizards from Puerto Rico, and find evidence that ultraviolet vision is important in their system of visual communication, which is based on displays consisting of motion patterns of the body and a colourful, ex-

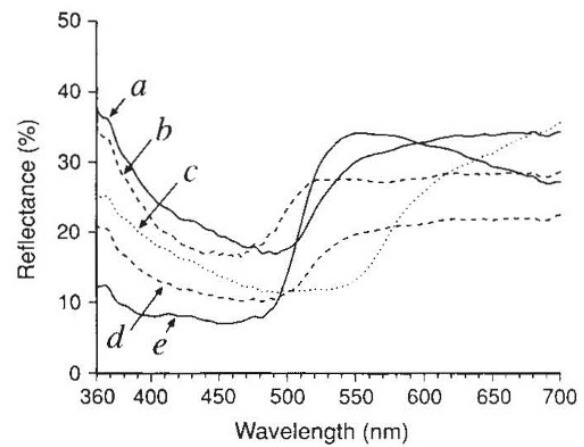

FIG. 1 Reflectance spectra (per cent reflectance compared to that from a magnesium carbonate white standard) from the dewlaps of a, Anolis krugi; b, A. cristatellus, c, A. pulchellus, $d, A$. gundlachi and $e, A$. evermanni.

pandable throat fan called the dewlap.

As part of a comprehensive study of the anoline retina (manuscript in preparation), we carried out microspectrophotometry and discovered that each of these five species has four classes of cones, with absorption maxima at about 565, 495, 450 and $365 \mathrm{~nm}$. The ultraviolet-sensitive cone $\left(\lambda_{\max }=365 \mathrm{~nm}\right)$ is always associated with a colourless oil droplet, which is transparent (optical density $<0.2$ ) down to the shortest wavelengths measured $(320 \mathrm{~nm})$, as were the lens and cornea. This is the first reported ultraviolet photoreceptor in any reptile, although earlier behavioural

\section{Scientific Correspondence}

Scientific Correspondence is a relatively informal section of Nature in which matters of general scientific interest, not necessarily those arising from papers appearing in Nature, are published. Because there is space to print only a small proportion of the letters received, priority is usually given according to general interest and topicality, to contributions of fewer than 500 words, and to contributions using simple language. studies have suggested that there is ultraviolet visual capacity in one turtle ${ }^{4}$ and one lizard ${ }^{5}$ species.

We measured the spectral reflectance of the dewlaps (Fig. 1), and found that two species exhibited a high degree of ultraviolet reflectance $(>35$ per cent), two exhibited low reflectance $(<25$ per cent $)$, and the third was intermediate. We photographed each dewlap in natural sunlight with an ultraviolet-sensitive video camera under two conditions: with a filter transmitting only in the near-ultraviolet and with a filter transmitting in the range of normal human vision (Fig. 2). As expected from Fig. 1, the dewlaps of Anolis cristatellus and $A$. krugi appeared very bright in ultraviolet light, whereas those of A. evermanni and A. gundlachi appeared dark. The dewlap of $A$. pulchellus has a distinct pattern in the ultraviolet - half dark, half bright - which explains why it appears to have an intermediate level of reflectance in Fig. 1. The photographs also revealed a bright spot at the corner of the mouth visible in all five species only when the mouth is open - a typical anoline threat display (Fig. 2).

There seems to be a relationship between the light conditions typical of the microhabitat of each species and the extent of dewlap ultraviolet reflectance. The three species with ultraviolet-bright dewlaps come from microhabitats which are often exposed to direct sunlight and/or blue $\mathrm{sky}^{6}$, in which there is a great deal of ultraviolet light ${ }^{7}$. The two species with ultraviolet-dark dewlaps inhabit the understorey of closed-canopy forest ${ }^{6,8}$, which contains little ultraviolet light ${ }^{7}$. Thus, in habitats where there is sufficient ultraviolet, an ultraviolet-reflective dewlap will appear bright and contrast sharply with the background vegetation, which reflects very little ultraviolet light. We believe that ultraviolet dewlap reflectance did not evolve (or was lost) in the two forest species because it would do little to enhance the visibility of the dewlap. The presence of ultraviolet visual receptors in the forest species may be due to phylogenetic inertia, or they may be for some other purpose (for example, prey detection near light gaps).

Our results strongly suggest that ultraviolet vision is involved in anoline communication because: (1) dewlaps are used almost exclusively in communication and hidden at other times; (2) only species

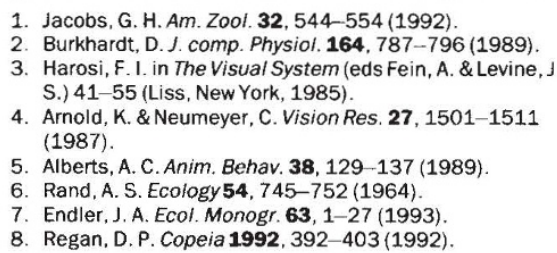

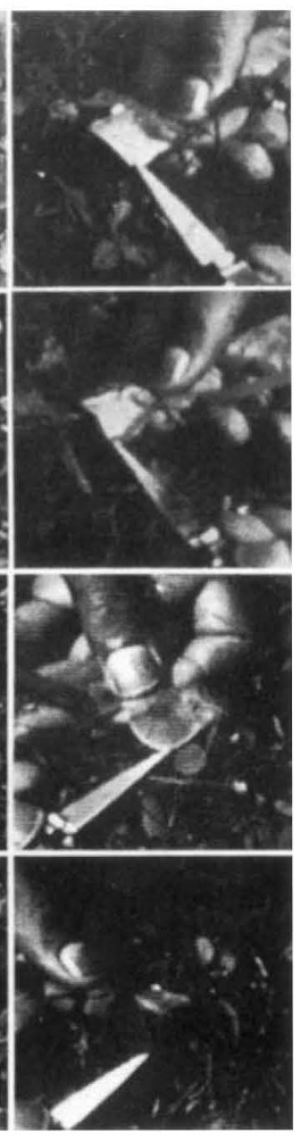

FIG. 2 An ultraviolet-enhanced video camera (CIDTEC) with a Flectan quartz reflecting lens (Nye Optical) was used to record the appearance of dewlaps under natural daylight conditions against a background of green grass. The first photograph in each row was made with filters passing light in the spectral range $400-680 \mathrm{~nm}$. The second photograph in each row shows the relative brightness in the nearultraviolet $(320-400 \mathrm{~nm})$ as isolated using a Hoya U360 filter plus an infrared blocker (Omega Optical). Contrast for the two spectral regions was equalized against a set of calibrated reflectance standards. The species shown are (top to bottom) A. cristatellus, $A$. pulchellus, $A$. gundlachi and $A$. evermanni. A. krugi (not shown) is similar in appearance to A. cristatellus

from ultraviolet-rich habitats possess ultraviolet-reflective dewlaps; and (3) only the dewlaps, and not the rest of the body, are ultraviolet-reflective.

Leo J. Fleishman

Department of Biological Sciences, Union College,

Schenectady,

New York 12308, USA

Ellis R. Loew

Department of Physiology,

College of Veterinary Medicine,

Cornell University,

Ithaca,

New York 14853, USA

Manuel Leal

Department of Biology,

University of Puerto Rico,

Rio Piedras,

Puerto Rico 00931-3360, USA 